Asian pearls

\title{
Validation of the Chinese version of Community Assessment of Psychic Experiences (CAPE) in an adolescent general population
}

\author{
Winifred Mark ${ }^{\mathrm{a}}$, Timothea Toulopoulou ${ }^{\mathrm{b}, \mathrm{c}, \mathrm{d}, \mathrm{e}, *}$ \\ ${ }^{a}$ Kwai Chung Hospital, Hospital Authority, Hong Kong Special Administrative Region \\ ${ }^{\mathrm{b}}$ Department of Psychology, The University of Hong Kong, Hong Kong Special Administrative Region \\ ${ }^{\mathrm{c}}$ The State Key Laboratory of Brain and Cognitive Sciences, The University of Hong Kong, Hong Kong Special Administrative Region \\ d Department of Psychology, Bilkent University, Ankara, Turkey \\ e Department of Basic and Clinical Neuroscience, the Institute of Psychiatry, Psychology and Neuroscience, King's College London, UK
}

\section{A R T I C L E I N F O}

\section{Article history:}

Received 11 May 2016

Received in revised form 5 January 2017

Accepted 16 January 2017

\section{Keywords:}

Community Assessment of Psychic

Experiences (CAPE)

Psychosis proneness

Schizophrenia

Chinese

Translation

Psychometrics

\begin{abstract}
A B S T R A C T
The Community Assessment of Psychic Experiences (CAPE) is a popular 42-item self-report assessment of psychosis proneness (PP) that has been widely-translated. However, there is as yet no validation of CAPE in non-Western languages. Here, we validated a Chinese translation of CAPE ("CAPE-C") in a young Chinese community sample. Factor analyses were employed in a sample of 660 individuals (mean age $=18.63$ ) to identify a culturally-sensitive factor structure for CAPE-C (Study 1 ). Since confirmatory factor analysis (CFA) suggested that CAPE-C did not follow the original factor structure, exploratory factor analysis and follow-up CFA were employed to establish an alternative structure, resulting in a 15-item "CAPE-C15" which retained a three-factor structure tapping positive, negative and depressive symptoms. To demonstrate the specificity of CAPE-C15 as a measure of PP, we conducted regression analyses to examine associations between CAPE-C15 dimensions and other measures of psychotic and depressive symptoms (Study 2). Results confirmed that CAPE-C15 dimensions showed specific associations with relevant symptom dimensions of other measures, but not with irrelevant ones. Finally, to aid interpretation of CAPE-C15 scores, Receiver Operating Characteristic analysis was conducted to establish a cut-off score that could indicate test-takers' need for clinical attention (Study 3). We found that a cut-off score of 8.18 on CAPE-C15 positive and negative symptom frequency and distress scores distinguished individuals whose PP was within normal ranges from those at psychometric high-risk (sensitivity: 78.6\%; specificity: $77.7 \%$ ). CAPE-C15 will likely prove relevant to researchers and healthcare providers who serve Chinese-speaking adolescents and young adults.
\end{abstract}

(c) 2017 Elsevier B.V. All rights reserved.

\section{Introduction}

Psychosis proneness (PP) refers to the extent to which an individual experiences psychotic-like experiences (PLEs) that do not reach clinical threshold for psychotic disorders. Despite being relatively prevalent in adolescence and young adulthood (van Os et al., 2009), such isolated symptoms are associated with an increased risk of debilitating psychiatric outcomes (Kelleher et al., 2012a, 2012b; Rössler et al., 2007; Werbeloff et al., 2012), and selfharm or suicide behaviors (Capra et al., 2015; Honings et al., 2016). As such, there is a need for accurate measurement of PP in research and clinical practice. One popular PP measure is the Community

\footnotetext{
* Corresponding author at: Department of Psychology, 6/F, the Centennial Campus, Pokfulam Road, Hong Kong Special Administrative Region.

E-mail address: timothea@hku.hk (T. Toulopoulou).
}

Assessment of Psychic Experiences (CAPE-42) (Stefanis et al., 2002), a 42-item self-report measure of frequency and distress of psychotic-like feelings, thoughts or mental experiences.

Seeing that CAPE-42 has not been validated in a non-Western language, here we report a validation study of a Chinese translation of CAPE-42 ("CAPE-C") in an adolescent and young adult community sample. Validation is important because translation can result in difference in item meaning, and different cultures may have different manifestations of PP. A late adolescent population was chosen since young people are at the highest risk to display PLEs (Laurens et al., 2008; Poulton et al., 2000), and to transition to a psychotic disorder (Harrop and Trower, 2001). By validating CAPE-C in adolescents and young adults, we hope to recast this aperture of vulnerability into a window of opportunity for early detection and intervention.

In a series of studies, we first statistically established a factor structure sensitive to young Chinese, resulting in a shortened 
questionnaire with 15 items ("CAPE-C15") (Study 1). We then compared CAPE-C15 to other established measures of psychotic and depression symptoms, in order to demonstrate the specificity of CAPE-C15 subscales in measuring positive psychotic symptoms, negative psychotic symptoms and depressive symptoms (Study 2). Finally, we established a cut-off score that could indicate testtakers' need for clinical attention (Study 3). The fruit of this effort is a comprehensive psychometric profile of CAPE-C15, which can facilitate its informed use by researcher and clinicians.

\section{Study 1: translation and factor structure of CAPE-C}

Study 1 concerned the psychometric robustness of CAPE-C, which is important because it informs whether scores obtained could be trusted. Psychometric robustness of a scale can be gleaned by its score "internal reliability" and "factor structure". Internal reliability concerns consistency of item scores (i.e., do CAPE-C items consistently measure the same construct?), while factor structure concerns scale composition (i.e., which items group together to form useful subscales in CAPE-C?).

To answer these questions, we first employed a confirmatory factor analysis (CFA) to test whether the original CAPE-42 factor structure model fitted our data. An acceptable model fit would mean that the scoring method of CAPE-42 could be directly adopted to generate meaningful CAPE-C scores. An unacceptable model fit, however, would mean that scores calculated by the original scoring method would not be interpretable for our data. Since model fit was not obtained in our data, exploratory factor analysis (EFA) was used to expose how best to restructure CAPE-C (e.g., by deleting questions, or regrouping questions into different subscales) in order to obtain meaningful scores. To make sure that scores generated from this alternative structure were reliable, Cronbach's alpha coefficients were calculated. Finally, to make sure that the structure suggested by EFA represented a good fit of Chinese data, follow-up CFA was conducted. Such a meticulous approach ensured that the factor structure reported here for CAPE-C was culturally-sensitive for a Chinese young population.

\subsection{Materials and methods}

\subsubsection{Participants}

669 Chinese adolescents and young adults, including 349 singletons (201 females, 148 males) and 320 twins or similarlyaged siblings (199 females, 118 males, 3 did not declare their gender), were recruited for the Twinsscan ${ }^{\text {China }}$ project, a twin and family study of PP in the Chinese population. Participants were recruited through twin registries, secondary schools and universities in five cities of The People's Republic of China: Hong Kong, Beijing, Nanjing, Qingdao and Guangzhou.

Singleton and sibling/twin samples were divided into three sub-samples for factor analyzes. In order to minimize familial covariance between participants in each sub-sample, siblings/ twins were assigned to separate subsamples in a pseudo-random fashion. We first generated randomized integers of "1", " 2 " and " 3 " using a computerized "random integer generator" as a group number for each participant. We then manually inspected the dataset to ensure that twins/siblings were assigned a group number that was different from that assigned to his/her co-twin/ sibling. The three sub-samples consisted of, respectively, 224 individuals (thereafter "Sample 1"), 250 individuals ("Sample 2"), and 186 individuals ("Sample 3").

\subsubsection{Instruments}

2.1.2.1. Community Assessment of Psychic Experiences (CAPE42). CAPE-42 contained three dimensions: the positive dimension (CAPE-pos; 20 items) tapped behaviors pertaining to reality distortion (e.g., "hear voices when you are alone"); the negative dimension (CAPE-neg; 14 items) tapped disruptions to normal behavior (e.g., "experience few or no emotions at important events"); and the depressive dimension (CAPE-dep; 8 items) included mainly cognitive symptoms of depression (e.g., "feel like a failure").

To ensure adequate translation of CAPE-42 from English to Chinese, translations by three Chinese-English bilingual speakers were back-translated by three other Chinese-English bilingual speakers blind to the study hypotheses and the English CAPE-42. Discrepancies in the forward and back translations were reviewed by the present investigators.

Administration and scoring of CAPE-C followed that of the original version (Stefanis et al., 2002). Participants rated their frequency of PLEs on a four-point Likert scale: "never" $=1$, "sometimes" $=2$, "often" $=3$, "nearly always" $=4$. Apart from an endorsement of "never", responders were asked to rate the degree of subjective distress associated with the psychotic experience from "not distressed" = 1, "a bit distressed" = 2, "quite distressed" = 3 to "very distressed" $=4$. To take into account partial nonresponse, a weighted score was calculated by sum of scores divided by the number of completed questions. Only frequency scores were used for analysis.

\subsection{Results}

\subsubsection{Descriptive statistics}

Participants' responses were screened for missing data, resulting in a final dataset of 660 participants (394 females, 263 males) with an average age of $18.63(S D=1.99)$. Samples 1,2 and 3 did not differ in proportion of participants from each data collection site, mean age of participants, gender ratio, and weighted total frequency scores on CAPE-C (Table 1). Hence, the randomization was considered successful.

Table 1

Demographic characteristics of randomized samples and internal reliability of CAPE-42 scale scores.

\begin{tabular}{|c|c|c|c|}
\hline & Sample 1 & Sample 2 & Sample 3 \\
\hline$N$ & 224 & 250 & 186 \\
\hline Mean (SD) age in years & $18.80(1.90)$ & $18.63(2.01)$ & $18.42(2.08)$ \\
\hline Ratio of participants collected from Beijing: Guangzhou: Hong Kong: Qingdao: Nanjing & 18: 15: 157: $28: 6$ & 23: $20: 170: 30: 7$ & 19: 17: 119: $26: 5$ \\
\hline Ratio of female: male & 132: 92 & 144: 105 & 118: 66 \\
\hline Ratio of singleton: sibling/twin & 127: 97 & 138: 112 & 84: 102 \\
\hline Weighted CAPE-C total frequency score & $1.89(.45)$ & $1.88(.37)$ & $1.82(.37)$ \\
\hline CAPE-C total frequency score Cronbach's alpha & 0.94 & 0.92 & 0.91 \\
\hline CAPE-C positive frequency score Cronbach's alpha & 0.87 & 0.85 & 0.82 \\
\hline CAPE-C negative frequency score Cronbach's alpha & 0.88 & 0.85 & 0.85 \\
\hline CAPE-C depressive frequency score Cronbach's alpha & 0.82 & 0.76 & 0.85 \\
\hline
\end{tabular}

Notes: CAPE-C = Chinese-translated Community Assessment of Psychic Experiences; SD = Standard deviation. 


\subsubsection{Internal reliability of CAPE-C}

Internal reliability of $\mathrm{CAPE}-\mathrm{C}$ scores was measured by Cronbach's alpha. Alpha values greater than 0.70 were considered to be acceptable for further analysis (Nunnally, 1978). Overall, frequency scores on CAPE-C (Table 1 ) showed "good" to "excellent" consistency across samples according to George and Mallery (2010).

\subsubsection{Confirmatory factor analysis of original CAPE-42 structure}

The internal structure of Sample 1 was fitted to that of the original CAPE-42 model with CFA using LISREL 9.1 (Joreskog and Sorbom, 2006). Of the 224 participants included, effective sample size (ESS) for analysis (excluding data of participants that completed less than $70 \%$ of CAPE-42 items or those with acquiescence response bias) was 217, yielding a participant:item ratio of 5.17. Questionnaire items were assumed to contribute information to only one latent factor in the model. Maximum likelihood estimation was used. The model produced goodness-offit indices as follows: RMSEA $=0.08, \mathrm{CFI}=0.87$, and $\mathrm{SRMR}=0.09$, which did not meet general criteria of model fit (i.e., RMSEA $\leq .07$, CFI $\geq .95$, SRMR < 0.08) (Hu and Bentler, 1999).

\subsubsection{Exploratory factor analysis for alternative factor structure}

Since goodness-of-fit indexes of the above CFA did not exceed established cut-offs, EFA was performed in SPSS 20.0 (IBM Corp., 2011 ) on Sample 2, to obtain a factor model that optimally accounts for the data in our Chinese population. Of the 250 individuals included, ESS was 212, yielding a participant:item ratio of 5.05 . Dimensions of PLEs were identified using EFA with principal axis factor extraction with Promax rotation for conservativeness (Osborne and Costello, 2009). The number of factors to be retained was determined by eigenvalues $>1$ and the scree plot (Fig. 1). EFA suggested a multiple-factor solution with 12 eigenvalues $>1$ and the scree plot suggested a three-factor solution.

The three-factor model accounted for $36.42 \%$ of the total variance. The first factor explained $23.69 \%$ of the variance, corresponding to items tapping depressive experiences. The second factor explained $7.32 \%$ of the variance, and comprised of items related to negative symptoms. The third factor explained $5.42 \%$ of the variance, and contained items concerning positive symptoms. The resultant model consisted of 15 items, hereafter known as "CAPE-C15" (Table 2).

\subsubsection{Internal reliability of CAPE-C15}

Cronbach's coefficient alpha was calculated for CAPE-C15 scores in Sample 2. Alpha value for CAPE-C15 total=.82 (15 items), positive scale $=.74$ ( 4 items), negative $=.69$ ( 5 items) and depressive scale $=.75$ ( 6 items).

\subsubsection{Confirmatory factor analysis of CAPE-C15}

The internal structure of Sample 3 was fitted to the CAPE-C15 factor structure, with a CFA using LISREL 9.1. Of the 186 participants included in Sample 3, ESS for analysis was 180, yielding a participant:item ratio of 12 . The model produced goodness-of-fit indices as follows: RMSEA=.04, CFI=.96, and SRMR=.061, which represented acceptable model fit (i.e., RMSEA $\leq .07, \mathrm{SRMR}<.08$, $\mathrm{CFI} \geq .95$ ) (Hu and Bentler, 1999). Hence, the CAPE-C15 factor structure was statistically robust.

\subsection{Discussion}

Recognizing the research and clinical values of CAPE-42, the present study was conducted to rectify the lack of validated Chinese translations in the current literature. We found that the original structure of CAPE-42 did not achieve acceptable model fit in our young Chinese community sample. Instead, a smaller set of 15 items was retained in CAPE-C15, clustered into three subscales corresponding to a positive, a negative, and a depressive dimension (i.e., a tri-dimensional model that mirrors the original CAPE-42 structure as proposed by Stefanis and colleagues). Our results corroborate our previous finding that item numbers and loadings tended to be different in translated versions of CAPE-42 (Mark and

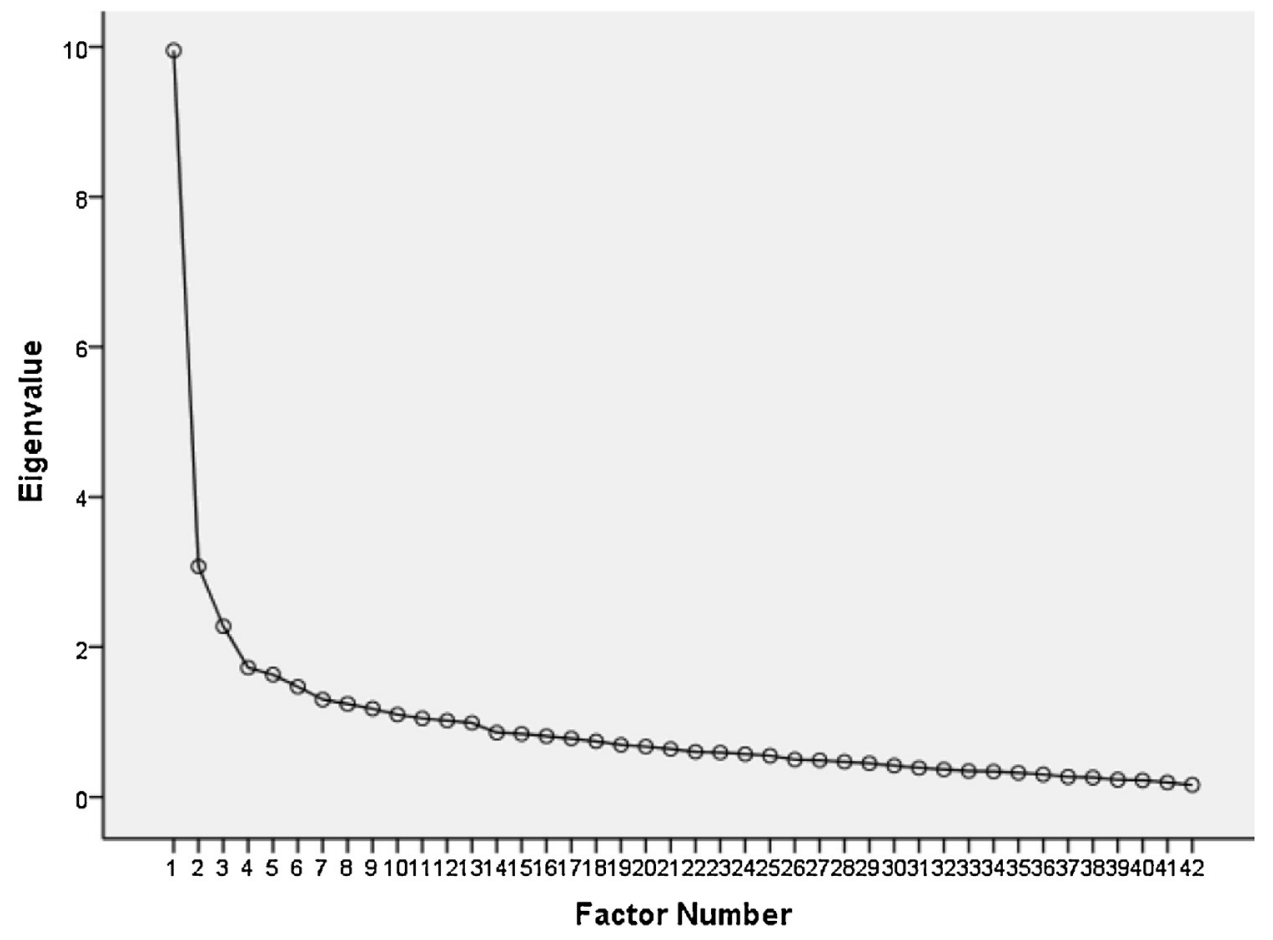

Fig. 1. Scree plot of exploratory factor analysis using Sample 2. The scree plot indicated three subscales. 
Table 2

Exploratory Factor Analysis of the Chinese CAPE-42 items in Sample 2.

\begin{tabular}{|c|c|c|c|c|}
\hline CAPE-42 Items & & Pos & Neg & Dep \\
\hline 01. & Feel sad & & & .908 \\
\hline 02. & People seem to drop hints about you or say things with a double meaning & & & \\
\hline 03. & Not a very animated person & & .799 & \\
\hline 04. & Not much of a talker when you are conversing with other people & & .527 & \\
\hline 05. & Things in magazines or on TV were written especially for you & & & \\
\hline 06. & Some people are not what they seem to be & & & \\
\hline 07. & Being persecuted in some way & & & \\
\hline 08. & Experience few or no emotions at important events & & .483 & \\
\hline 09. & Pessimistic about everything & & & \\
\hline 10. & Conspiracy against you & .369 & & \\
\hline 11. & Destined to be someone very important & & & \\
\hline 12. & No future for you & & & \\
\hline 13. & You are a very special or unusual person & & & \\
\hline 14. & Do not want to live anymore & & & .533 \\
\hline 15. & Communicate telepathically & & & \\
\hline 16. & No interest to be with other people & & & \\
\hline 17. & Electrical devices such as computers can influence the way you think & & & \\
\hline 18. & Lacking in motivation to do things & & & .407 \\
\hline 19. & Cry about nothing & & & .539 \\
\hline 20. & Believe in the power of witchcraft, voodoo or the occult & & & \\
\hline 21. & Lacking in energy & & .712 & \\
\hline 22. & People look at you oddly because of your appearance & & & \\
\hline 23. & Mind is empty & & & \\
\hline 24. & Thoughts in your head are being taken away from you & & & \\
\hline 25. & Spending all your days doing nothing & & & \\
\hline 26. & Thoughts in your head are not your own & & & \\
\hline 27. & Feelings are lacking in intensity & & & \\
\hline 28. & Thoughts so vivid that you were worried other people would hear them & & & \\
\hline 29. & Lacking in spontaneity & & & \\
\hline 30. & Hear your own thoughts being echoed back to you & & & \\
\hline 31. & Under the control of some force or power other than yourself & & & \\
\hline 32. & Your emotions are blunted & & & \\
\hline 33. & Hear voices when you are alone & & & \\
\hline 34. & Hear voices talking to each other when you are alone & .515 & & \\
\hline 35. & You are neglecting your appearance or personal hygiene & & & \\
\hline 36. & You can never get things done & & & .506 \\
\hline 37. & You have only few hobbies or interests & & .440 & \\
\hline 38. & Feel guilty & & & \\
\hline 39. & Feel like a failure & & & .385 \\
\hline 40. & Feel tense & & & \\
\hline 41. & A double has taken the place of a family member, friend or acquaintance & .640 & & \\
\hline 42. & See objects, people or animals that other people cannot see & .905 & & \\
\hline
\end{tabular}

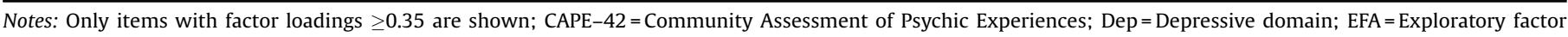
analysis; Neg = Negative domain; Pos = Positive domain.

Toulopoulou, 2016), further reinforcing the need for proper validation of translations.

Deleted items tended to have low communalities (i.e., these items explained too small an amount of variance in the retained factors to be preserved) or were assessed by similar items that had been retained. In spite of the greatly reduced number of items, the CAPE-pos subscale structure previously found in our meta-analysis of CAPE-pos factor analytic studies (Mark and Toulopoulou, 2016) was retained in CAPE-C15. Specifically, items pertaining to "Bizarre experiences", "Delusional ideations" and "Perceptual anomalies" could be found in the positive subscale of CAPE-C15 while items pertaining to "Social withdrawal", "Affective flattening" and "Avolition" could be found in the negative subscale of CAPE-C15. It is, however, worthy of note that two items originally proposed to be in CAPE-neg were loaded onto the depressive factor in CAPE-C15. This is congruent with the view that negative symptoms show phenomenological similarities to depressive symptoms (Murali and Kumar, 2008; Newcomer et al., 1990; Sax et al., 1996). Longitudinal studies suggest that the two could be differentiated by their temporal characteristics: depressive symptoms varied more over time while negative symptoms tended to be more stable and trait-like (Häfner et al., 2005). However, since CAPE was designed to be a self-report of lifetime psychotic experience, it might be difficult to differentiate the lack of motivation to establish regular activities such as hobbies (negative symptom) and lack of motivation to pursue established hobbies (depression) without further information on the time course of the symptom.

One limitation of our study is the relatively small sample size relative to the population of China. However, our factor analytic studies all yielded participant:item ratios that lied in or exceeded the suggested range of 3 to 6 (Cattell, 1978). Hence, our results are statistically valid. A related caveat is that CAPE produces skewed data and low rates of endorsement on certain items in the general population, as is the case for most measurements of subclinical psychopathology. This raises the issue of low variation in item responses due to small sample size. However, we do not think this could explain our results since further item response analysis of the data set indicated that most items achieved at least moderate item discrimination. Another caveat is that the score reliability in CAPE-C15 was lower than in the initial version. However, the possibility that this relates to the lower number of scale items could not be excluded (Streiner et al., 2014). To conclude, Study 1 presented preliminary data that CAPE-C15 is suitable for indexing $\mathrm{PP}$ in a young Chinese population. Further replication studies will serve to confirm this. 


\section{Study 2: convergent-divergent validity of CAPE-C}

To be a useful assessment tool in research and clinical settings, CAPE-C should only measure what it supposedly measures, and not measure irrelevant constructs. This is known as "convergentdivergent validity". To examine the convergent-divergent validity of CAPE-C15, CAPE-pos and CAPE-neg symptom dimensions of CAPE-C15 need to be tested against another PP instrument. The questions of interest are: does CAPE-pos, but not CAPE-neg, correlate with assessments of positive symptoms? Conversely, does CAPE-neg, but not CAPE-pos, correlate with assessments of negative symptoms? This method is known as "cross-validation".

Previous research has suggested that CAPE-pos and CAPE-neg had convergent-discriminant validity (Konings et al., 2006). However, cross-validation of CAPE-neg and CAPE-dep was often neglected in the literature. Given that CAPE-dep was especially added to CAPE-42 in order to discriminate between negative and depressive symptoms (Stefanis et al., 2002), it is important to establish whether CAPE-neg and CAPE-dep indeed specifically measured negative PP symptoms and depressive symptoms respectively.

In this study, cross-validation was carried out with CAPE-C15 on the one hand; and an interview-based schizotypy measure "Structured Interview for Schizotypy-Revised (SIS-R)" as well as a self-report depression measure in "Symptom Checklist 90 (SCL90)" on the other. SIS-R was selected so that subtle PLEs that might be missed by self-report could be captured. The depression subscale of SCL-90 was selected for comparison with CAPE-dep since SCL-90 has been repeatedly validated in Chinese populations (Chen and Li, 2003; Feng and Zhang, 2001; Hu, 2006; Liu and Zhang, 2004), and has separate norms for Chinese adolescents (Liu and Zhang, 2004). It was hypothesized that CAPE-pos and CAPEneg would show independent and specific associations with the relevant symptom dimension in SIS-R or SCL-90 (convergent validity), and show non-significant associations with the irrelevant symptom dimension (divergent validity). In order to test the assumption that CAPE-C15 leads to more a valid estimation of positive symptoms, negative symptoms, and depression symptoms than the 42-item CAPE-C in a Chinese population sample, a direct comparison of convergent-divergent validity of the CAPE-C15 and CAPE-C was conducted.

\subsection{Materials and methods}

\subsubsection{Participants}

The sample included 212 participants recruited for Twinsscan $^{\text {China }}$ (71 male, 139 female, 2 did not declare their gender). Only siblings/twins collected in Hong Kong and Beijing were included in the convergent-divergent analysis for CAPE-C15, since only these groups completed CAPE, SIS-R and SCL-90.

\subsubsection{Instruments}

3.1.2.1. Chinese-translated Community Assessment of Psychic Experiences (CAPE-C)/15-item Community Assessment of Psychic Experiences (CAPE-C15). All participants completed the CAPE-C as described in Study 1. Separate analyses were done to compare CAPE-C items and CAPE-C15 items. CAPE-C15 contained four items describing positive PLEs of hallucination and persecutory beliefs; five items describing negative symptoms of affective flattening, social withdrawal and apathy; and six items describing depressive experiences including behavioral and cognitive aspects. Administration and scoring procedures were identical to that in Study 1 . Score reliabilities of CAPE-C frequency subscale were 0.93 for total score, 0.86 for CAPE-pos, 0.85 for CAPE-neg, and 0.78 for CAPE-dep. Score reliabilities of CAPE-C15 frequency subscale were
0.86 for total score, 0.74 for CAPE-pos, 0.67 for CAPE-neg, and 0.74 for CAPE-dep.

3.1.2.2. Structured Interview for Schizotypy-Revised (SIS-R). A short version of the SIS-R consisting of 11 symptoms and 4 signs was used (Collin et al., 2011; Lataster et al., 2014; van den Berg et al., 2013). The shortened SIS-R could be subdivided into a positive symptom dimension tapping referential thinking, suspiciousness, magical ideation, illusions, psychotic symptoms, and derealization/depersonalization; and a negative symptom dimension tapping social isolation, introversion, hypersensitivity, restricted affect, thought disturbances (e.g., tangentiality), loosening of associations, poverty of speech and odd/eccentric behavior. To reduce interviewers' idiosyncratic judgments, Vollema and Ormel (2000) explicitly defined the criteria for each symptom and sign, which researchers assessed with standardized questions tapping frequency, duration and degree of conviction or observation. Based on such information, researchers gave an overall rating of severity on a four-point Likert scale ("absent" $=0$, "mild" = 1 , "moderate" $=2$ and "severe" $=3$ ).

3.1.2.3. Symptom Checklist 90 (SCL-90). The SCL-90 (Derogatis, 1983 ) is a self-report questionnaire assessing psychopathology, including somatization, obsessive-compulsive, interpersonal sensitivity, depression, anxiety, hostility, phobic anxiety, paranoid ideation, and psychoticism symptoms. Participants scored the 90 items on a five-point Likert scale based on distress ("none" $=1$, "a little bit" $=2$, "moderate" $=3$, "quite a bit" $=4$, "extreme" $=5$ ). A weighted score was computed for each subscale by adding all scores divided by the number of items in each subscale. Only the depression subscale was used in this analysis. The depression subscale contained symptoms consistent with the clinical diagnosis of a major depressive episode in DSM-5 (American Psychological Association, 2013). The internal reliability of the SCL-90 depression subscale (13 items) in this sample was acceptable: Cronbach's alpha $=0.90$.

\subsection{Results}

\subsubsection{Descriptive statistics}

Participants' responses were screened for missing data. The final sample consisted of 196 participants (64 male, 130 female; two participants did not declared their gender) with a mean age of 17.76 $(S D=1.97)$. The mean, standard deviation and standard errors of each variable of interest are presented in Table 3.

\subsubsection{Convergent-divergent validity}

Since the data was hierarchical in nature due to siblingship, multilevel regressions were performed with maximum likelihood estimation to examine associations between dimensions of CAPEC15 on the one hand, and the SIS-R dimensions or SCL-90 depression scale on the other.

Table 3

Mean and standard deviation of CAPE-C15, SIS-R and SCL-90 scores.

\begin{tabular}{lll}
\hline & Mean & SD \\
\hline CAPE-C15 positive dimension frequency & 1.59 & 0.62 \\
CAPE-C15 negative dimension frequency & 2.09 & 0.58 \\
CAPE-C15 depressive dimension frequency & 1.95 & 0.52 \\
SIS-R positive dimension & 3.55 & 2.75 \\
SIS-R negative dimension & 3.09 & 2.14 \\
SCL-90 depression subscale & 1.66 & 0.62 \\
\hline
\end{tabular}

Notes: CAPE-C15 = 15-item Chinese Community Assessment of Psychic Experiences; SCL-90=Symptoms Checklist 90; SIS-R=Structured Interview for Schizotypy, Revised. 
Multilevel regression with CAPE-C15 positive and negative subscale scores as predictors and SIS-R positive domain score the outcome showed that CAPE-C15 positive domain significantly predicted SIS-R positive dimension scores $(ß=1.70,95 \% \mathrm{CI}=1.07$, $2.33, p<.001$ ). The CAPE-C15 negative domain did not significantly predict SIS-R positive symptom domain scores ( $\beta=0.47,95 \%$ $\mathrm{CI}=-0.20,1.13, p=.17)$. Hence, only CAPE-pos, but not CAPE-neg, of CAPE-C15 was associated with SIS-R positive symptom dimension.

Multilevel regression with CAPE-C15 positive and negative subscale scores as predictors and SIS-R negative domain score as the outcome showed that CAPE-C15 negative domain significantly predicted SIS-R negative dimension scores $(B=1.03,95 \% \mathrm{CI}=0.50$, $1.57, p<.001)$. The CAPE-C15 positive domain did not significantly predict SIS-R negative symptom domain scores $(\beta=.34,95 \%$ $\mathrm{CI}=-0.17,0.85, p=.19$ ). Hence, only CAPE-neg, but not CAPE-pos, of CAPE-C15 was associated with SIS-R negative symptom dimension.

Multilevel regression with CAPE-C15 negative and depressive subscale scores as predictors and SCL-90 depression subscale scores as the outcome showed that CAPE-C15 depressive domain significantly predicted SCL-90 depression subscale scores $(ß=0.48$, $95 \% \mathrm{CI}=0.29,0.67, p<.001)$. The CAPE-C15 negative domain did not significantly predict SIS-R depression symptom domain scores ( $ß=0.13,95 \% \mathrm{CI}=-0.05,0.30, p=.15)$. Hence, only CAPE-dep, but not CAPE-neg, of CAPE-C15 was associated with SCL-90 depression dimension.

Furthermore, to test the assumption that CAPE-C15 leads to more a valid estimation of positive symptoms, negative symptoms, and depression symptoms than the 42-item CAPE-C, multilevel regressions were performed with maximum likelihood estimation to examine associations between dimensions of the 42-item CAPE$\mathrm{C}$ on the one hand, and the SIS-R dimensions or SCL-90 depression scale on the other. Multilevel regression with CAPE-C positive and negative subscale scores as predictors and SIS-R positive domain score as the outcome showed that CAPE-C positive domain significantly predicted SIS-R positive dimension scores $(\beta=1.98$, $95 \% \mathrm{CI}=0.89,3.08, p<.001$ ). The CAPE-C negative domain did not significantly predict SIS-R positive symptom domain scores ( $ß=.83,95 \% \mathrm{CI}=-.16,1.83, p=.10$ ). Hence, only CAPE-pos, but not CAPE-neg, of the 42-item CAPE-C was associated with SIS-R positive symptom dimension.

Multilevel regression with CAPE-C positive and negative subscale scores as predictors and SIS-R negative domain score as the outcome showed that CAPE-C negative domain significantly predicted SIS-R negative dimension scores $(B=1.52,95 \% \mathrm{CI}=0.71$, $2.33, p<.001$ ). The CAPE-C positive domain did not significantly predict SIS-R negative symptom domain scores $(B=.10,95 \%$ $\mathrm{CI}=-.81,1.01, p=.83$ ). Hence, only CAPE-neg, but not CAPE-pos, of the 42-item CAPE-C was associated with SIS-R negative symptom dimension.

Multilevel regression with CAPE- $C$ negative and depressive subscale scores as predictors and SCL-90 depression subscale scores as the outcome showed that CAPE-C15 depressive domain significantly predicted SCL-90 depression subscale scores $(ß=0.33$, $95 \% \mathrm{CI}=0.11,0.55, p=.003)$. The CAPE-C negative domain also significantly predicted SIS-R depression symptom domain scores ( $\beta=0.39,95 \% C I=0.18,0.61, p<.001$ ). Hence, both CAPE-dep and CAPE-neg of the 42-item CAPE-C were associated with SCL-90 depression dimension.

In summary, the discriminant validity of CAPE-C15 negative and depressive scales are superior to that of the 42-item CAPE-C.

\subsection{Discussion}

Study 2 was conducted to examine the convergent-divergent validity of CAPE-C15. Consistent with our hypotheses, results confirmed independent significant associations with relevant subscales in SIS-R and SCL-90, and non-significant associations with irrelevant subscales. This showed that subscales of CAPE-C15 had convergent-divergent validity, supporting the individual subscales as specific measures of positive PP, negative PP and depressive symptoms. In addition, this study also found that CAPE-neg of CAPE-C15 could discriminate between negative and depressive symptoms better than that of the 42-item CAPE-C. This is important because negative symptoms show phenomenological similarities to depressive symptoms (Murali and Kumar, 2008; Newcomer et al., 1990; Sax et al., 1996), and must be accurately classified to avoid misassessment. Taken together, CAPE-C15 could measure positive, negative and depressive symptoms robustly, showing promise as a screening tool for PP.

\section{Study 3: CAPE-C15 as a screening tool: ROC analysis}

Having established CAPE-C15's psychometric properties, the next step is to consider its application in research and clinical settings. To do so, researchers and clinicians must know at which point a score on CAPE-C15 indicated elevated PP. One way to approach this is to conduct a Receiver Operating Characteristic (ROC) analysis. ROC analysis works by using an "index test" (i.e., the test of interest; in our case, CAPE-C15) to predict classification of individuals who did or did not warrant clinical attention as determined by a "golden standard" (i.e., a validated measure or clinician diagnosis). This results in a CAPEC15 cut-off score that classifies individuals into those who experience PLEs but are not distressed by them (prevalence \%\%; van Os et al., 2009), and psychometric high-risk individuals who report PLEs that do not cross clinical threshold for a diagnosis of psychotic disorder, but who nonetheless experience distress and may deserve clinical attention (prevalence $=4 \%$; van Os et al., 2009).

CAPE cut-off scores from ROC analyses have been previously reported in help-seeking populations. Research showed that using the Dutch CAPE-42 as an adjunct to clinical interviewing improved detection of first episode psychosis (FEP) in new referrals at a mental health service clinic (Boonstra et al., 2009). That data yielded a cut-off of 50 (out of 80) on either the frequency or distress dimension of CAPE-pos, which provided a sensitivity of $77.5 \%$ and a specificity of $70.5 \%$. This means that, using a raw score cut-off of 50 on either CAPE-pos frequency or CAPE-pos distress ruled in $77.5 \%$ of individuals who have FEP, while ruling out $70.5 \%$ of individuals who did not have FEP. Similarly, there is evidence that the German CAPE-42 was useful in detecting individuals at ultra-high risk for psychosis in a help-seeking clinical population. Two cut-off points were suggested for a weighted CAPE-pos frequency and distress score: 3.20 (sensitivity: 67\%; specificity: $73 \%$ ) and 2.80 (sensitivity: $83 \%$; specificity: $49 \%$ ) (Mossaheb et al., 2012).

Study 3 aimed to establish a cut-off score for CAPE-C15. In contrast to aforementioned studies that employed help-seeking populations, we were interested in using CAPE to screen for psychometric high-risk populations in a general population. Given the association of PP with clinical outcomes (Kelleher et al., 2012a; Kelleher et al., 2012b; Rössler et al., 2007; Werbeloff et al., 2012), such a cut-off score would facilitate early detection in non-clinical settings (e.g., schools) for referral to primary healthcare. A "CAPE-C15 PLE frequency and distress score" composed of the frequency and distress scores of CAPE-pos and CAPE-neg were used as the index scores, and were compared to a gold standard as defined by the SCL-90 psychoticism subscale score. 


\subsection{Materials and methods}

\subsubsection{Participants}

401 singleton participants (161 male, 238 female; 2 did not declare his/her gender) with a mean age of $19.68(\mathrm{SD}=1.54)$ were included. They were recruited through universities in Hong Kong.

\subsubsection{Instruments}

4.1.2.1. Chinese-translated Community Assessment of Psychic Experiences (CAPE-C)/15-item Chinese-translated Community Assessment of Psychic Experiences (CAPE-C15). All participants completed CAPE-C as described in Study 1. CAPE-C and CAPE-C15 were the index tests of separate analyses. A weighted CAPE-C/ CAPE-C15 PLE frequency and distress score was calculated by summing frequency and distress scores for each item of the positive and negative scales, divided by the number of questions completed. Score reliability was acceptable: Cronbach's alpha was .86 for the CAPE-C positive frequency and distress score and .84 for the CAPE- $C$ negative frequency and distress score. Cronbach's alpha was .88 for the CAPE-C15 positive frequency and distress score and .72 for the CAPE-C15 negative frequency and distress score.

4.1.2.2. Symptom Checklist 90 (SCL-90). The SCL-90 (Derogatis, 1983) was chosen as the golden standard due to the availability of well-established norms for Chinese adolescents (Chen and $\mathrm{Li}$, 2003; Feng and Zhang, 2001; Hu, 2006; Liu and Zhang, 2004). Only the psychoticism subscale was used in this analysis. The psychoticism subscale taps experiences associated with the psychosis continuum, from social withdrawal to hallucinations and delusions. In accordance with guidelines from the Beijing Suicide and Research Prevention Center, a cut-off of 3 was used to indicate an elevated level of symptomatology that warranted clinical attention in Chinese populations. SCL-90 psychoticism subscale scores in our sample were acceptable: Cronbach's alpha $=.83$.

\subsection{Results}

\subsubsection{Descriptive statistics}

Participants' responses were screened for missing data on either CAPE-C weighted PLE frequency and distress scores or SCL90 psychoticism subscale. Five participants were excluded for incomplete SCL-90 psychoticism subscale scores. The final sample included 396 participants (160 male and 236 female) with mean age of 19.68 ( $S D=1.54)$. Fourteen $(3.54 \%)$ participants were "SCL90-positive" (i.e., above cut-off) and 382 (96.46\%) were "SCL-90negative" (i.e., below cut-off). Mean weighted CAPE-C15 PLE frequency and distress score was $6.67(\mathrm{SD}=2.15)$, while that of SCL90 psychoticism subscale was $1.56(\mathrm{SD}=.58)$.

\subsubsection{Receiver operating characteristic analysis}

ROC analysis was conducted in SPSS 20.0 (IBM Corp., 2011). The area under the ROC curve is the measure of the effectiveness and validity of the index test (Hajian-Tilaki, 2013), which was .817 $(\mathrm{SE}=.065)$ in our analysis, confirming CAPE-C15 PLE frequency and distress scores as a "good" predictor (Swets et al., 2000). The optimal cut-off score was determined by the Youden criteria (Youden, 1950), which represented the optimal statistical cut-off corresponding to a combination of high sensitivity and specificity. A cut-off of 8.18 resulted in a sensitivity of $78.6 \%$ and a specificity of $77.7 \%$.

ROC analysis of the 42-item CAPE-C resulted in an area under curve of .828 ( $\mathrm{SE}=.055)$ in our analysis, confirming CAPE-C PLE frequency and distress scores as a "good" predictor (Swets et al.,
2000). Using the Youden criteria revealed that an optimal cut-off of 9.14 resulted in a sensitivity of $92.9 \%$ and a specificity of $74.1 \%$.

\subsection{Discussion}

Our study is the first to date to establish a cut-off score for differentiating individuals with normal-range PP from psychometric high-risk individuals who might warrant clinical attention, in the general population. ROC analysis suggested a cut-off score of 8.18 for the weighted CAPE-C15 PLE frequency and distress scores. Comparing it to a cut-off of 9.14 for CAPE-C (sensitivity $=92.9 \%$; specificity $=74.1 \%$ ), one could see that including all 42 questions only increased the optimal cut-off by $\sim 1$ point and resulted in a drop in specificity, which supports item reduction. CAPE-C15 may be used in routine student health checkups for screening individuals who are experiencing psychotic symptoms and who may be distressed by them.

\section{Conclusion}

When conducting cross-cultural replication research, the significance of employing properly translated and validated questionnaires cannot be over-emphasized. Given that any data collected is only as robust as the instrument that collected them, a poorly-translated and validated questionnaire leads inevitably to corrupt data, which in turn results in spurious and unsubstantiated conclusions. Our Chinese CAPE-C15 enriches the predominately Western CAPE literature, and could facilitate PP research as well as early detection and intervention in the Chinese population. Our results are relevant to the young population targeted in the study, but further studies are needed to confirm the extent to which they generalize to older populations.

The strength of this study lies in the meticulous translation and validation procedures. This is the first translation validation report of CAPE with comprehensive documentation of score reliability, factor structure, convergent-divergent validity, as well as a cut-off score that indicated clinical needs in a community sample. However, the present study was not immune to problems inherent to self-report measures, including social desirability and over- or under-reporting of symptoms. Possible cultural influences on social desirability further complicate the task of test development (Green, 2009). That being said, CAPE-C15's psychometric robustness and user-friendliness renders it an accessible tool for quick self-evaluation and self-referral, or for risk assessment for referral to primary healthcare in non-clinical settings (e.g., schools). All in all, our three studies supported the use of CAPE-C15 in research and community samples of Chinese adolescent and young adult populations.

\section{References}

American Psychological Association, 2013. Diagnostic and Statistical Manual of Mental Disorders: DSM-5. American Psychological Association.

Boonstra, N., Wunderink, L., Systema, S., Wiersma, D., 2009. Improving detection of first episode psychosis by mental health care services using self-report questionnaire. Early Interv. Psychiatry 3 (4), 289-295.

Capra, C., Kavanagh, D.J., Hides, L., Scott, J.G., 2015. Subtypes of psychotic-like experiences are differentially associated with suicidal ideation, plans and attempts in young adults. Psychiatry Res. 228 (3), 894-898.

Cattell, R.B., 1978. The Scientific Use of Factor Analysis in Behavioral and Life Sciences. Plenum Press, New York.

Chen, S., Li, L., 2003. Re-testing reliability, validity and norm applicatility of SCL-90. Chin. J. Nerv. Mental Dis. 29 (5), 323-327.

Collin, G., Hulshoff Pol, H.E., Haijma, S.V., Cahn, W., Kahn, R.S., van den Heuvel, M.P. 2011. Impaired cerebellar functional connectivity in schizophrenia patients and their healthy siblings. Front. Psychiatry 2, 73.

Derogatis, L.R., 1983. SCL-90-R: Administration, Scoring, and Procedures Manual II. Clinical Psychometric Research Baltimore.

Feng, Z.Z., Zhang, D.J., 2001. Study on the validity of the symptom check-list-90 of Chinese version. Acta Acad. Med. Militaris Tertiae 23, 481-483. 
George, D., Mallery, M., 2010. SPSS for Windows Step by Step: A Simple Guide and Reference, 17.0 Update, 10th ed. Pearson, Boston.

Green, B.A., 2009. Culture and Mental Health: Sociocultural Influences, Theory, and Practice. Wiley-Blackwell, Oxford.

Häfner, H., Maurer, K., Trendler, G., an der Heiden, W., Schmidt, M., Könnecke, R. 2005. Schizophrenia and depression: challenging the paradigm of two separate diseases-a controlled study of schizophrenia, depression and healthy controls. Schiz. Res. 77 (1), 11-24. doi:http://dx.doi.org/10.1016/j.schres.2005.01.004.

Hajian-Tilaki, K., 2013. Receiver operating characteristic (ROC) curve analysis for medical diagnostic test evaluation. Casp. J. Intern. Med. 4 (2), 627-635.

Harrop, C., Trower, P., 2001. Why does schizophrenia develop at late adolescence? Clin. Psychol. Rev. 21 (2), 241-265.

Honings, S., Drukker, M., Groen, R., van Os, J., 2016. Psychotic experiences and risk of self-injurious behaviour in the general population: a systematic review and meta-analysis. Psychol. Med. 46 (02), 237-251.

Hu, L.T., Bentler, P.M., 1999. Cutoff criteria for fit indexes in covariance structure analysis: conventional criteria versus new alternatives. Struct. Equ. Model. 6 (1) $1-55$.

Hu, S.L., 2006. Confirmatory factor analysis and norms comparison of SCL-90 in middle school students. Stud. Psychol. Behav. 4 (2), 114-119.

IBM Corp, 2011. IBM SPSS Statistics for Windows, Version 20.0. IBM Corp., Armonk, NY.

Joreskog, K.G., Sorbom, D., 2006. LISREL for Windows, 9.1 ed. Scientific Software International, Inc, Skokie, IL.

Kelleher, I., Keeley, H., Corcoran, P., Lynch, F., Fitzpatrick, C., Devlin, N., Molloy, C. Roddy, S., Clarke, M.C., Harley, M., Arseneault, L., Wasserman, C., Carli, V., Sarchiapone, M., Hoven, C., Wasserman, D., Cannon, M., 2012a. Clinicopathological significance of psychotic experiences in non-psychotic young people: evidence from four population-based studies. Br. J. Psychiatry 201 (1), 26-32.

Kelleher, I., Lynch, F., Harley, M., et al., 2012b. Psychotic symptoms in adolescence index risk for suicidal behavior: findings from 2 population-based case-contro clinical interview studies. Arch. Gen. Psychiatry 69 (12), 1277-1283.

Konings, M., Bak, M., Hanssen, M., van Os, J., Krabbendam, L., 2006. Validity and reliability of the CAPE: a self-report instrument for the measurement of psychotic experiences in the general population. Acta Psychiatr. Scand. 114 (1), 55-61.

Lataster, T., Verweij, K., Viechtbauer, W., 2014. Effect of illness expression and liability on familial associations of clinical and subclinical psychosis phenotypes. Acta Psychiatr. Scand. 129 (1), 44-53.

Laurens, K.R., West, S.A., Murray, R.M., Hodgins, S., 2008. Psychotic-like experiences and other antecedents of schizophrenia in children aged 9-12 years: a comparison of ethnic and migrant groups in the United Kingdom. Psychol. Med. 38 (8), 1103-1111.

Liu, H., Zhang, J., 2004. Norm of symptom checklist (SCL-90) in chinese middle school students. Chin. Mental Health Journal 18 (2), 88-90.

Mark, W., Toulopoulou, T., 2016. Psychometric properties of community assessment of psychic experiences: review and meta-analyses. Schizophr. Bull. 42 (1), 34 44.
Mossaheb, N., Becker, J., Schaefer, M.R., Klier, C.M., Schloegelhofer, M., Papageorgiou, K., Amminger, G.P., 2012. The Community Assessment of Psychic Experience (CAPE) questionnaire as a screening-instrument in the detection of individuals at ultra-high risk for psychosis. Schizophr. Res. 141, 210-214.

Murali, T., Kumar, R., 2008. Symptoms of depression in schizophrenia. Indian J. Med. Res. 127, 516-518.

Newcomer, J.W., Faustman, W.O., Yeh, W., Csernansky, J.G., 1990. Distinguishing depression and negative symptoms in unmedicated patients with schizophrenia. Psychiatry Res. 31 (3), 243-250.

Nunnally, J.C., 1978. Psychometric Theory, 2nd ed. McGraw-Hill, New York.

Osborne, J.W., Costello, A., 2009. Best practices in exploratory factor analysis: four recommendations for getting the most from your analysis. Pan-Pac. Manage. Rev. 12 (2), 131-146.

Poulton, R., Caspi, A., Moffitt, T.E., Cannon, M., Murray, R., Harrington, H., 2000. Children's self-reported psychotic symptoms and adult schizophreniform disorder: a 15-year longitudinal study. Arch. Gen. Psychiatry 57 (11), 1053-1058.

Rössler, W., Riecher-Rossler, A., Angst, J., Murray, R., Gamma, A., Eich, D., van Os, J., Gross, V.A., 2007. Psychotic experiences in the general population: a twentyyear prospective community study. Schizophr. Res. 92 (1-3), 1-14.

Sax, K.W., Strakowski, S.M., Keck Jr., P.E., Upadhyaya, V.H., West, S.A., McElroy, S.L., 1996. Relationships among negative, positive, and depressive symptoms in schizophrenia and psychotic depression. Br. J. Psychiatry: J. Mental Sci. 168 (1), 68-71.

Stefanis, N.C., Hanssen, M., Smirnis, N.K., Avramopoulos, D.A., Evdokimidis, I.K., Stefanis, C.N., Verdoux, H., van Os, J., 2002. Evidence that three dimensions of psychosis have a distribution in the general population. Psyhcol. Med. 32, 347358.

Streiner, D.L., Norman, G.R., Cairney, J., 2014. Health Measurement Scales: a Practical Guide to Their Development and Use. Oxford university press.

Swets, J.A., Dawes, R.M., Monahan, J., 2000. Psychological science can improve diagnostic decisions. Psychol. Sci. Public Interest 1, 1-26.

Vollema, M.G., Ormel, J., 2000. The reliability of the structured interview for schizotypy-revised. Schizophr. Bull. 26 (3), 619-629.

Werbeloff, N., Drukker, M., Dohrenwend, B.P., Levav, I., Yoffe, R., van Os, J., Davidson, M., Weiser, M., 2012. Self-reported attenuated psychotic symptoms as forerunners of severe mental disorders later in life. Arch. Gen. Psychiatry 69 (5), 467-475.

van Os, J., Linscott, R.J., Myin-Germeys, I., Delespaul, P., Krabbendam, L., 2009. A systematic review and meta-analysis of the psychosis continuum: evidence for a psychosis proneness-persistence-impairment model of psychotic disorder. Psychol. Med. 39 (2), 179-195.

van den Berg, S.M., Paap, M.C.S., Derks, E.M., 2013. Genetic Risk and Outcome of Psychosis (GROUP) investigators, Using multidimensional modeling to combine self-report symptoms with clinical judgment of schizotypy. Psychiatry Res. 206, 75-80.

Youden, W.J., 1950. Index for rating diagnostic tests. Cancer 3, 32-35. 